\title{
Gold nanoparticle-based theranostics: disease diagnostics and treatment using a single nanomaterial
}

This article was published in the following Dove Press journal:

Nanobiosensors in Disease Diagnosis

5 May 2015

Number of times this article has been viewed

\author{
Raquel Vinhas ${ }^{1,2, *}$ \\ Milton Cordeiro ${ }^{1,3, *}$ \\ Fábio Ferreira Carlos ${ }^{1,4}$ \\ Soraia Mendo ${ }^{1,2}$ \\ Alexandra R Fernandes ${ }^{2}$ \\ Sara Figueiredo' \\ Pedro V Baptista'
}

'Nanomedicine@FCT, CIGMH

(Centro de Investigação em Genética

Molecular Humana), UCIBIO,

Departamento de Ciências da Vida,

Faculdade de Ciências e Tecnologia,

Universidade Nova de Lisboa,

Campus da Caparica, Caparica,

Portugal; ${ }^{2} \mathrm{UCIBIO}$ (Unidade de

Ciências Biomoleculares Aplicadas),

Departamento de Ciências da Vida,

Faculdade de Ciências e Tecnologia,

Universidade Nova de Lisboa, Campus

da Caparica, Caparica, Portugal;

${ }^{3}$ REQUIMTE, Departamento de

Química, Faculdade de Ciências e

Tecnologia, Universidade Nova de

Lisboa, Campus da Caparica, Caparica,

Portugal; ${ }^{4}$ STABVIDA, Investigação e

Serviços em Ciências Biológicas, Lda,

Madan Parque, Caparica, Portugal

*These authors contributed equally to this work

Correspondence: Pedro V Baptista Nanomedicine@FCT, CIGMH, UCIBIO, Departamento de Ciências da Vida, Faculdade de Ciências e Tecnologia, Universidade Nova de Lisboa, Campus da Caparica, 2829-516 Caparica, Portugal Tel/Fax +35I 212948530

Email pmvb@fct.unl.pt

\begin{abstract}
Nanotheranostics takes advantage of nanotechnology-based systems in order to diagnose and treat a specific disease. This approach is particularly relevant for personalized medicine, allowing the detection of a disease at an early stage, to direct a suitable therapy toward the target tissue based on the molecular profile of the altered phenotype, subsequently facilitating disease monitoring and following treatment. A tailored strategy also enables to reduce the off-target effects associated with universal treatments and improve the safety profile of a given treatment. The unique optical properties of gold nanoparticles, their ease of surface modification, and high surface-to-volume ratio have made them central players in this area. By combining imaging, targeting, and therapeutic agents in a single vehicle, these nanoconjugates are (ought to be) an important tool in the clinics. In this review, the multifunctionality of gold nanoparticles as theranostics agents will be highlighted, as well as the requirements before the translation of these nanoplatforms into routine clinical practice.
\end{abstract}

Keywords: nanotheranostics, nanomaterials, gold nanoparticle, theranostics, cancer, nanomedicine

\section{Introduction}

Theranostics is aimed toward the development of platforms capable of combining diagnostic and therapeutic agents. ${ }^{1,2}$ These innovative systems combined into single platforms should help overcome the troublesome differences in biodistribution and specificity of imaging molecules and therapeutic drugs. ${ }^{3,4}$ Theranostic approaches have been proposed for the management of several diseases/disorders, in particular for cancer diagnostics and therapeutics. Cancer therapy greatly relies on chemotherapy and radiotherapy in which most anticancer drugs are essentially taken up by cells with high proliferative rate, a characteristic of cancer cells. However, extensive proliferation is not exclusive for tumor cells, and normal tissue also suffers from chemotherapeutic action, leading to severe side effects. ${ }^{5-7}$ The efficacy of conventional therapies is also often limited by the natural tendency of cells to evade therapy through mutational events that lead to multidrug resistance, poor drug penetration into tumor tissues, and serious damage to normal tissues..$^{8-10}$

Conventional therapy toward heterogeneous diseases is rarely targeted directly toward a specific cell type or tissue. In fact, the administration of a specific treatment to a subpopulation of patients in accordance to their molecular profile and risk of adverse effects is believed to be a more effective clinical model for improved patient outcome. By selecting patients for targeted clinical trials, based on biomarker 
identification, personalized medicine is expected to reduce health costs caused by unnecessary treatment.

To overcome these issues, alternative strategies have been proposed in which nanomedicine plays a key role, such as for innovative drug delivery systems that increase the bioavailability and concentration of anticancer drugs at target site, resulting in improved therapeutic index. ${ }^{6,711}$ Combination of diagnostic and therapeutic entities into a single delivery vector using nanotechnology - nanotheranostics - constitutes a step forward for tailored early disease detection, medical treatment, and disease prevention. ${ }^{12,13}$ These nanoformulations must ascertain whether an individual carries a specific disease biomarker and release the appropriate therapeutic dose in a kinetically regulated manner. The design of such a system ought to include the possibility of targeted delivery ${ }^{14,15}$ (to minimize off-target accumulation and, therefore, minimize side effects), and the release of the imaging/therapeutic molecule should be precisely triggered in the specific cellular environment. ${ }^{16,17}$ The developed platform should allow a combination of different effector molecules (eg, stabilizer, therapeutic agent, targeting and imaging moieties) for maximization of theranostic effect. ${ }^{18,19}$

Here, we shall address options for surface functionalization of gold nanoparticles (AuNPs) toward therapy (section "Surface functionalization of AuNPs toward therapy"), and focus on the use of AuNPs as theranostic agents, ie, imaging purposes (section "AuNPs for imaging purposes") and therapy, eg, from drug and gene delivery vehicles to photothermal ablation agents (section "Gold nanoparticles: from conventional drug and gene delivery vehicles to photothermal ablation agents"). Current trends relating to the translation of theranostic nanomaterials from the laboratory to the clinics shall be discussed in section "Theranostic nanomaterials: from the laboratory to the clinics?".

Several nanotheranostics platforms have been presented over the past decade, namely, metal and silica nanoparticles (NPs), liposomes, and composite NPs (Table 1). ${ }^{6,11,20,21}$ These platforms allow visualization and monitoring of the route taken by the formulation, providing information about delivery kinetics, intra-organ and/or intratumor distribution, and drug efficacy. Using a single NP, it is possible to tune therapy while simultaneously providing for real-time monitoring of disease progression. ${ }^{1,22}$ Traditional methodologies such as $\mathrm{X}$-ray computed tomography (CT) and magnetic resonance imaging (MRI) do not display sufficient sensitivity to diagnose the asymptomatic phases of a disease, ${ }^{23,24}$ and owing to current levels of sensitivity, numerous patients entail extended unnecessary treatments. A theranostic strategy might allow treatment termination once the biomarker levels are undetectable.
AuNPs have great potential to be explored as a multifunctional tool for a wide range of biological applications..$^{25-27}$ Their unique optical properties make them excellent noninvasive imaging agents, adding to their high surface-tovolume ratio that allows them to be loaded with a plethora of molecules, such as targeting/imaging moieties and therapeutic agents (Figure 1). By integrating targeting, diagnostics, treatment, and monitoring, AuNPs overcome several constraints associated with conventional methods of diagnostics and treatment, ensuring the codelivery of imaging and therapeutic molecules at the same site, with real-time monitoring of their effects. ${ }^{14,18,28}$ Also, targeted molecular imaging and delivery of the therapeutic moiety, with enhanced retention at the target sites, allow reduction of the nanoconjugate concentrations in circulation and therefore reduce systemic toxicity. ${ }^{19,29}$

AuNPs may work as a multimodal imaging tool by loading them with two or more contrast agents (fluorophores, MRI agents, photoacoustic agents, surface-enhanced Raman spectroscopy [SERS] reporters) to improve disease characterization and avoiding a repeated challenge on the patient immune system. ${ }^{30}$ This is also valid for therapeutic agents: one can take advantage of the synergistic effects of different treatments (effective drug, photothermal therapy, and/or antisense DNA) by combining them onto a single AuNP.

This review will focus on nanotheranostics using a single agent, addressing specifically AuNPs, the need for this new model, and its advantages over conventional methodologies. A long way has been paved since the first theranostic agent - radiopharmaceutical iodine - was used for thyroid disease in the 1940s ${ }^{31}$ We are now facing clinical trials of AuNPs for treatment of solid tumors: Aurimune ${ }^{\circledR}$ (PEGylated colloidal gold particles; Phase I) (CytImmune Sciences, Rockville, MD, USA) and AuroLase ${ }^{\circledR}$ (gold nanoshell; Phase I) (Nanospectra Biosciences, Inc., Houston, TX, USA). We will also address some bottlenecks that still need to be circumvented before the most promising agents can be effectively translated to the clinical practice, eg, in vivo efficacy, stability, and toxicity.

\section{Surface functionalization of AuNPs toward therapy}

The chemical functionality and reactivity of AuNPs may be customized through surface functionalization, which modulates the solubility, stability, charge effect of the nanocomplex, and interaction with other molecules. ${ }^{14,32-36}$ One of the most used approaches has been the use of thiolated biomolecules to easily functionalize the particle surface. ${ }^{33,37}$ Such plasticity allows the modification and design of the AuNPs' 
Table I Advantages and disadvantages of different nanotheranostics platforms

\begin{tabular}{|c|c|c|}
\hline Type of nanoparticle & Advantages & Disadvantages \\
\hline \multirow[t]{6}{*}{ Mesoporous silica NPs } & Large surface area & In vivo toxicity \\
\hline & Stability & \\
\hline & Biocompatibility & \\
\hline & Controllable porosity & \\
\hline & Surface reactivity and ease of functionalization & \\
\hline & Biodegradability & \\
\hline \multirow[t]{4}{*}{ Magnetic NPs } & Large surface area & Poor colloidal stability \\
\hline & Small size allows longer circulation and tissue penetration & Limited biocompatibility and cytotoxicity \\
\hline & Controlled clustering & Nonbiodegradability \\
\hline & Multimodal applications (targeting, diagnostics, and therapy) & \\
\hline \multirow[t]{7}{*}{ Gold NPs } & Large surface area & High costs for large-scale production \\
\hline & Low hydrodynamic mean size & Lack of standard protocols for translation into the clinics \\
\hline & Multimodal applications (targeting, diagnostics, and therapy) & Nonbiodegradability \\
\hline & Suitable for photodynamic therapy & \\
\hline & Scaffold for additional agents & \\
\hline & Ease of surface modification & \\
\hline & Stability and biocompatibility & \\
\hline \multirow{9}{*}{ Liposomes } & Flexibility of synthesis, modification, and formulation & Low drug-loading efficiency \\
\hline & $\begin{array}{l}\text { Ease of conjugation and functionalization with targeting, } \\
\text { contrast agents, probes, and fluorophores }\end{array}$ & Batch-to-batch variation in manufacturing \\
\hline & Rapid cellular uptake and well-characterized cell & High production cost \\
\hline & internalization mechanism & \\
\hline & Controlled release & Limited instability and leakage of loaded materials \\
\hline & Scaffold for additional agents & Low solubility \\
\hline & Low immunogenicity & Rapid clearance \\
\hline & Biocompatibility & \\
\hline & Commercially available products & \\
\hline \multirow[t]{4}{*}{ Dendrimers } & Very precise size and shape controllability & Nonspecific cytotoxicity \\
\hline & Water solubility and biocompatibility & Limited release of the associated bioactives \\
\hline & Elicit negligible immune response & Rapid clearance \\
\hline & Easy electrostatic interaction with nucleic acids & \\
\hline \multirow[t]{4}{*}{ Hydrogels } & Selective surface modification & High production cost \\
\hline & High degree of porosity and high loading capacity & Instability \\
\hline & Controlled and sustained release into target tissues & \\
\hline & Biocompatibility and biodegradability & \\
\hline \multirow[t]{7}{*}{ Quantum dots } & High photo- and chemical stability & Toxicity \\
\hline & Size- and structure-based tunable emission & Difficult surface modification (thiol groups) \\
\hline & High molar extinction coefficient & Particle aggregation, degradation, and removal \\
\hline & Low hydrodynamic mean size & \\
\hline & Scaffold for additional agents & \\
\hline & Potential for synergistic application in diagnostics and & \\
\hline & therapeutic applications. Suitable for photodynamic therapy & \\
\hline \multirow[t]{6}{*}{ Carbon nanotubes } & Large surface area & Nonbiodegradable \\
\hline & Ultrahigh functionalization and loading capacities & Toxicity (oxidative stress) \\
\hline & High penetration capacity to biological barriers & Lack of a standard protocol to prepare high-purity \\
\hline & Scaffold for additional agents & carbon nanotubes in large scale \\
\hline & Imaging probe on its own with high spatial resolution & \\
\hline & Suitable for photodynamic therapy & \\
\hline
\end{tabular}

Note: Adapted with permission from Draz M, Fang B, Zhang P, et al. Nanoparticle-mediated systemic delivery of siRNA for treatment of cancers and viral infections. Theranostics. 2014;4(9): 1023-1030.12

Abbreviation: NPs, nanoparticles.

surface chemistry, thus granting control over the circulation half-time and systemic clearance of these structures in the body, ${ }^{38}$ and allows the modification and tailoring of bioavailability, immunogenicity, and preferential accumulation site. ${ }^{39}$ There is a wide range of stabilizers available, such as thiolated biomolecules, polymers, dendrimers, phosphine, that may simultaneously act as therapeutic moieties (eg, microRNA, DNA, peptides, and antibodies). Polyethylene glycol (PEG) is one of the most widely used stabilizer molecules able to increase AuNPs' circulation half-life and cellular uptake, with 


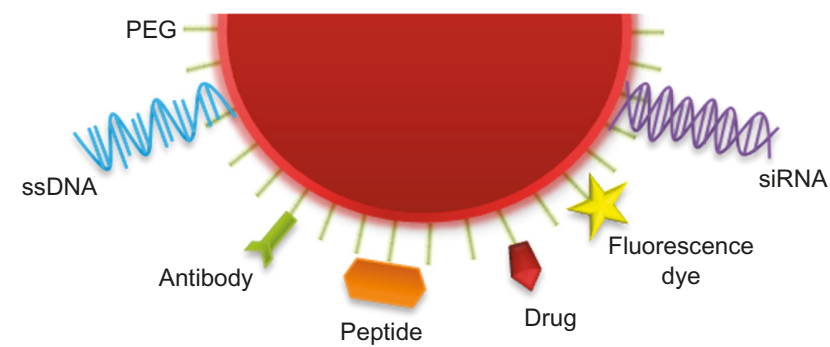

Figure I Representation of an AuNP for theranostics.

Note: AuNPs loaded with a plethora of moieties allow AuNPs to act as molecular sensors for disease diagnostics, as therapeutic agents by themselves due to their photothermal conversion capacity, and also as vehicles for delivering other effective therapeutics and imaging agents to specific locations.

Abbreviations: AuNP, gold nanoparticle; PEG, polyethylene glycol.

reduced immunogenicity. ${ }^{40}$ Bifunctional PEGs with a thiol molecule in one extremity and a different functional group on the other extremity (eg, amine, carboxylic, biotin, azide) have also been used to allow further functionalization with other biomolecules (Table 2).

The surface of an AuNP can also be modified simply using ionic coupling methods, in which the ligand exhibits an opposite charge to that of the AuNP or of the capping agent. ${ }^{41,42}$ Ionic coupling procedures are quicker and do not require preactivation of molecules but originate more unstable constructs, and control over the biomolecule orientation is hindered..$^{43}$ The widely known biotin-streptavidin interaction can also be used for the surface modification of AuNPs. Although noncovalent, the functionalization of the AuNP with avidin or biotin usually requires a covalent immobilization of these molecules (either by direct functionalization using a thiolated molecule or through EDC coupling). Hydrophobic interactions, while not being covalent interactions, usually require a covalent attachment using a thiol coupling. ${ }^{44}$

Considering the ease of surface modification, coupled with their optical properties, AuNPs are good candidates for the development of theranostics systems. For instance, doxorubicin (DOX) can be loaded to PEG-coated hollow gold nanospheres (HAuNS) both inside and outside HAuNS, increasing the DOX payload compared to solid AuNPs. Using near-infrared (NIR) laser, DOX is released in the region of interest, inducing cancer cell death. ${ }^{45}$ Nanorods functionalized with DOX may be further conjugated to a cRGD peptide for tumor targeting and $\mathrm{pH}$-sensitive controlled drug release. ${ }^{46}$ Increased cytotoxicity of PEG-AuNPs functionalized with oxaliplatin has been demonstrated, when compared to free oxaliplatin, in A549 lung epithelial cancer cells and several colon cancer cell lines (HCT116, HCT15, HT29, and RKO), possibly due to enhanced cellular uptake. ${ }^{47}$

\section{Gold nanoparticles as theranostic agents}

Smartly designed NPs have already made their way to the clinics, eg, Doxil ${ }^{\circledR}$ (Janssen Biotech Inc, Horsham, PA, USA), Abraxane ${ }^{\circledR}$ (Celgene Corporation, Berkeley Heights, NJ, USA) - therapeutic application; Resovis ${ }^{\circledR}$ (Bayer Schering Pharma AG, Berlin, Germany) and Feridex ${ }^{\circledR}$ (Bayer Healthcare Pharmaceuticals, Wayne, NJ, USA) - imaging agents, but the development of an equivalent able to combine both strategies is still underway. Indeed, several platforms have already been proposed integrating therapeutic strategies (ie, chemo-, genetic-, immunotherapy, photothermal ablation) and imaging agents to monitor NP fate, eg, MRI, CT, and photoacoustic tomography (PAT). ${ }^{48-50}$

Nanomaterials, such as AuNPs, exhibit remarkably different properties from the bulk material. Owing to the spatial confinement of the conduction electrons, unique optical properties arise (localized surface plasmon resonance [LSPR]) with strong impact on sensing and therapeutic applications. ${ }^{51-54}$ The intensity and frequency at which the LSPR occurs may be tailored through the variation of the metal composition, size, shape, and the dielectric surrounding. ${ }^{55,56}$ The light extinction induced by the LSPR

Table 2 Common covalent immobilization strategies for AuNP functionalization

\begin{tabular}{|c|c|c|c|c|}
\hline $\begin{array}{l}\text { Coupling } \\
\text { reaction }\end{array}$ & $\begin{array}{l}\text { Functional group } \\
\text { at AuNP surface }\end{array}$ & $\begin{array}{l}\text { Functional group } \\
\text { on ligand }\end{array}$ & Linkers/spacers & $\begin{array}{l}\text { Examples of } \\
\text { biomolecules coupled }\end{array}$ \\
\hline Thiol & Direct coupling & Thiol & - & $\begin{array}{l}\text { Thiolated DNA }{ }^{28,64} \\
\text { Thiolated PEG } 116 \\
\text { Thiolated fluorophores }\end{array}$ \\
\hline $\mathrm{EDC} / \mathrm{NHS}$ & $\begin{array}{l}\text { Carboxylic/phosphate } \\
\text { or amine }\end{array}$ & $\begin{array}{l}\text { Amine or carboxylic/ } \\
\text { phosphate }\end{array}$ & $\begin{array}{l}\text { Bifunctional PEG/DNA chain } \\
\text { harboring a thiol and a(n) amine/ } \\
\text { carboxylic group }\end{array}$ & $\begin{array}{l}\text { Proteins }^{117} \\
\text { Antibodies }^{14} \\
\text { Fluorophores }^{118}\end{array}$ \\
\hline Maleimide & Thiol/maleimide & Maleimide/thiol & $\begin{array}{l}\text { Homo-bifunctional thiolated PEG or } \\
\text { hetero-bifunctional PEG harboring } \\
\text { a maleimide }\end{array}$ & $\begin{array}{l}\text { Peptides } 119 \\
\text { DNA }^{120} \\
\text { Chemotherapeutic agents }\end{array}$ \\
\hline
\end{tabular}

Abbreviations: AuNP, gold nanoparticle; EDC, I-ethyl-3-(3-dimethylaminopropyl)carbodiimide; NHS, N-hydroxysuccinimide; PEG, polyethylene glycol. 
comprises both absorption and scattering processes. ${ }^{57}$ The scattering component of the extinction band is proportional to the particle size, while the absorption component is inversely proportional to the particle size. ${ }^{53}$ AuNPs' spectral behavior may also be explored in other regions of the spectrum. When an AuNP absorbs light, it can decay (in a nonradiative manner) to a lower energy level through the generation of heat. This particular phenomenon can be applied in photothermal therapy. Not only the LSPR is highly tunable, but also its extinction coefficient for laser absorption can be five orders of magnitude higher than those of conventional organic dyes used in photothermal procedures. ${ }^{53}$ Moreover, AuNPs are known to be resistant to photobleaching. ${ }^{58}$

Although in an early stage of technological development, AuNPs-based diagnostic and therapeutic approaches have already been proposed to carry a drug or imaging agents directly targeted to a specific tissue and/or cell type. Generally, particulates between $10 \mathrm{~nm}$ and $60 \mathrm{~nm}$ in diameter tend to passively accumulate in tumor tissues due to the enhanced permeability and retention (EPR). ${ }^{59} \mathrm{EPR}$ occurs due to changes to tumors' endothelia, where the leaky vasculature allows extravasation of NPs to the extracellular milieu and decreased lymphatic drainage results in local accumulation. Yet, although NPs tend to naturally accumulate in tumors, functionalization allows proper recognition of selective cellular target - active targeting.

\section{AuNPs for imaging purposes}

AuNPs are extremely versatile since they may act as an imaging probe on their own and/or be easily functionalized with suitable imaging agent, overcoming some of the technique's limitations. In fact, the idea of using multiple modalities has become popular, and recently, hybrid imaging instruments have become available, with the first to appear being a fusion of positron emission tomography (PET)/CT instrument, followed by PET/MRI, and, more recently, ultrasound/CT. These techniques may clearly benefit from multimodality, since a single agent could avoid the administration of multiple doses. ${ }^{30,60}$ The choice of imaging modality must be carefully considered since each one has its own advantages and limitations - eg, modalities with the highest sensitivity have relatively poor resolution. ${ }^{60}$ Improvement in these systems might allow the observation of tissues not only on its basic anatomic configuration but also on the molecular level. Moreover, real-time noninvasive monitoring of theranostic NPs enables a rapid decision on whether the treatment regimen is effective in a given patient. Herein, the focus shall be on AuNPs, and for further insights into other systems, please refer to additional reading. ${ }^{61-63}$

\section{AuNPs as fluorescence modulators}

AuNPs and other noble metal NPs are known to modulate fluorescence of fluorophores in their vicinity, ${ }^{64-66}$ including quenching due to energy transfer or charge transfer. As the distance between a NP and a fluorophore increases, quenching by the NP decreases. Modulation of fluorescence has been used for the development of biosensing approaches and tracking of nanoformulations in living systems. One such example suitable for theranostics is the so-called gold nanobeacons (Au nanobeacons), ie, AuNPs functionalized with an effector ssDNA in a hairpin configuration tagged with a suitable fluorophore. ${ }^{14,28}$ Upon hybridization to the specific target (eg, mRNA or miRNA), the hairpin opens, quenching by the AuNP ceases to exert its influence, and fluorescence is observed, thus allowing to track the silencing effect within cells and tissues. This simple nanotheranostics platform may be easily applied to any RNA sequence (Figure 2).

Traditional fluorophore quenching may be substituted by SERS. ${ }^{67}$ In fact, noble metal NPs, such as gold and silver, are known to promote SERS of adsorbed dyes on NP surfaces, which has been widely used for biosensing in complex media. ${ }^{68,69}$

These optical properties (high extinction coefficient with possibility of heat generation or scattered light, fluorescence modulation, and SERS) may be explored in a wide range of theranostics applications, which may also profit from the ease of AuNP surface modification with no observable toxicity. ${ }^{19}$

\section{Computed tomography and magnetic resonance imaging}

CT is often the first approach for the detection of the region of pathology within tissues and organs. As for MRI, CT does not have the needed sensitivity to target molecular events or even to diagnose abnormal cellular events, which require contrast agents, such as gadolinium $(\mathrm{Gd})$ in MRI and iodine contrast in $\mathrm{CT}^{70,71}$ Although routinely used in the clinics, iodinated and Gd-based (to a lower extent) contrast agents exhibit side effects, namely, CT-associated life-threatening contrast-induced nephropathy and MRI-associated nephrogenic systemic fibrosis. ${ }^{71}$ For instance, contrast enhancement in CT is usually based on low-molecular-weight compounds that show rapid clearance, where AuNPs appear as an interesting alternative. ${ }^{70,72-74}$ In fact, acetylated dendrimerentrapped gold nanoparticles (AuDENPs) were used for 


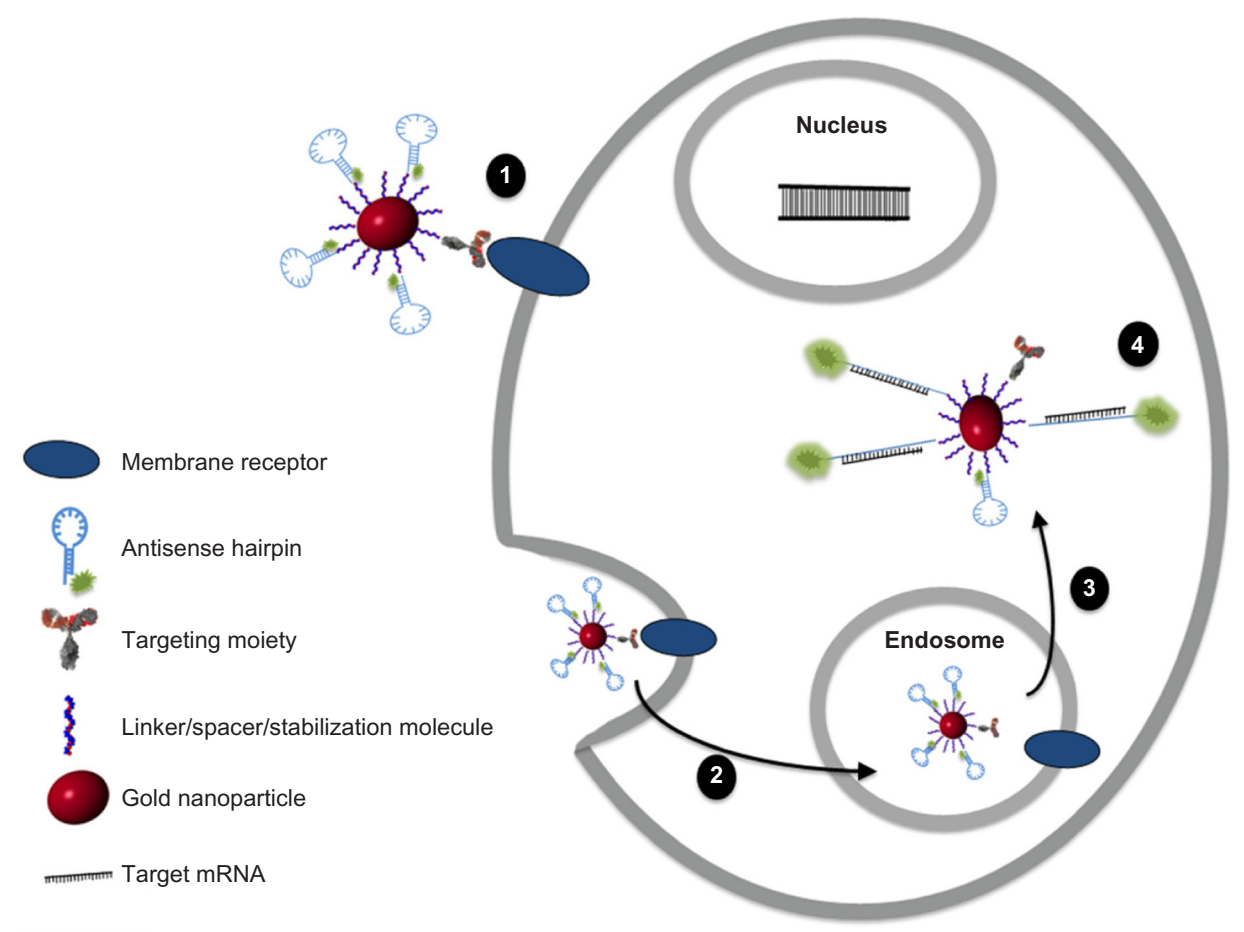

Figure 2 Au nanobeacons as a theranostic approach for specific gene silencing with real-time monitoring of the inhibition process.

Notes: 1) Target cell recognition, 2) Au nanobeacon internalization, 3) endosome escape, 4) gene silencing with concomitant enhancement of fluorophore emission. Abbreviation: Au, gold.

in vitro and in vivo CT imaging of a human lung adenocarcinoma cell line (SPC-A1 cells). Intratumoral and intraperitoneal administration of acetylated AuDENPs allowed the detection of cancer cells under X-ray in xenograft tumor models. In vitro assays show that acetylated AuDENPs are biocompatible and do not affect cell viability, making them good CT contrast agents. ${ }^{75}$

Effective examples of AuNPs for MRI analysis include an innovative vector for improving MRI contrast agents currently used for clinical diagnosis. ${ }^{72,76}$ Moreover, Gdloaded AuDENPs can be used for dual-mode CT/MRI. ${ }^{77}$ For instance, Alric et $\mathrm{al}^{72}$ functionalized AuNPs with diethylenetriaminepentaacetic acid (DTPA) to chelate $\mathrm{Gd}$ ions $\left(\mathrm{Gd}^{3+}\right)$. A total of $50 \mathrm{Gd}$ ions per particle increases the system's stability, allowing the application as contrast agent for both MRI and X-ray CT in vivo. Owing to their size, these nanoconjugates can also be used as sensitizers for radiotherapy. ${ }^{72}$ Similarly, nanoconjugates formed by silica layer-coated gold nanorods to which folic acid (FA) was covalently anchored (AuNR-SiO ${ }_{2}$-FA) were shown to be selectively taken up by MGC803 cells, subsequently enhancing their sensitivity to $\mathrm{X}$-ray radiation. This way, these particulates may become a good candidate for X-ray CT imaging associated to tumor targeting. These same nanoconjugates could be eventually used for photothermal therapy. ${ }^{49}$
Another example is the Co@Pt-AuNP platform that presents strong magnetism and high stability, resulting in a good MRI contrast agent to monitor the progression of $A \beta$ protofibrils involved in Alzheimer's. ${ }^{78}$

\section{Photoacoustic tomography}

AuNPs may also be used in PAT, a noninvasive modality combining high sensitivity of optical imaging with high resolution of ultrasound imaging. ${ }^{79}$ Contrast agents for PAT are photoabsorbing agents that, when excited at their resonance wavelength, convert light into heat (photothermal conversion) correlated to the generation of acoustic waves (due to thermoelastic expansion), which can then be detected by an ultrasound transducer. ${ }^{80}$ PAT has been slowly overcoming the limited resolutions of techniques dependent solely on ultrasonic diffraction. When used in the NIR of the spectrum, PAT is not constrained by the optical diffusion limit, which allows the reconstruction of images with improved spatial resolution, deep penetration depth, and excellent image contrast when compared to conventional optical imaging. ${ }^{81}$ Gold nanorods, due to their cylindrical shape, present characteristic SPR toward the NIR, and are of extreme use for $\mathrm{PAT}^{82,83}$ PAT molecular imaging in deep tissue has also been improved by using reporter-gene products. ${ }^{84-86}$ Recently, Taruttis et $\mathrm{al}^{87}$ demonstrated real-time imaging of siRNA in 
liposome-AuNR hybrids within tumors through multispectral optoacoustic tomography, an upgrade of PAT.

\section{Gold nanoparticles: from conventional drug and gene delivery vehicles to photothermal ablation agents}

AuNPs' high surface area makes them proper systems for efficient vectorization of high payloads of drugs,${ }^{45}$ including siRNAs or microRNAs. ${ }^{18,28,88} \mathrm{~A}$ wide range of molecules may be used for active targeting toward effective nanotheranostics, from hormones to cytokines, antibodies, and peptides (Table 3).

Another efficient way to reduce side effects is via systems that release their payload in a unique environment, as response to either internal or external stimuli - smart agents (triggered release). These systems may respond to changes in the $\mathrm{pH}$ or even to specific protease activity within the tumor surroundings..$^{89,90}$ However, owing to AuNPs' intrinsic characteristics, light and heat have been the most commonly explored actuator of drug release. ${ }^{91,92}$ As mentioned earlier, when AuNPs absorb light, it can be dissipated as heat, which may be used for the controlled release of drugs functionalized or adsorbed to the AuNPs' surface..$^{93}$ In addition, the photothermal effect might increase blood perfusion in the tumor region, further increasing the delivery of therapeutics. ${ }^{94}$ AuNPs' shape and size can be tuned at will (eg, nanospheres, nanorods, nanocages, or nanoshells), whose spectral behavior is directly correlated with a shift in the LSPR to the NIR region (650-900 nm), thus allowing deeper tissue penetration of light. Indeed, AuNP-mediated hyperthermia shows particular promise in animal studies, and early clinical testing is currently underway. ${ }^{95}$

Combination of imaging platforms with hyperthermia is currently being developed in which a single particle is used to image tumors and later accurately and efficiently ablate them. Yushen et al developed a microcapsule of AuNP-poly(lactic acid) with surface deposition of graphene oxide. The assembly was used for in vivo NIR photothermal therapy, suitable as contrast agent for both ultrasound and $\mathrm{CT}$ imaging. Together, AuNPs and graphene oxide coating allowed improved photothermal conversion, with the final outcome being total tumor remission due to the performed laser ablation treatment. ${ }^{73}$ However, the challenge of using gold nanorods in this type of therapy is the observation that after an intense and prolonged period under laser illumination, gold nanorods might lose stability, being reshaped to gold nanospheres, thus losing response to NIR frequencies. ${ }^{96}$
Although gold nanorods tend to be considered the "gold standard" in photothermal therapy, colloidal gold nanospheres have more frequently been used as theranostic agents. Their main disadvantage is the absorbance peak, situated in the visible region, whereas the low toxicity of nanospheres is one of the biggest advantages, since geometry plays a considerable role in acute cell toxicity. Hyperthermia success of AuNPs is determined by several factors, namely, sufficient depth penetration (up to $1 \mathrm{~cm}$ in the NIR) and low energy absorption by healthy tissues (to reduce collateral damage). For example, Melancon et al conjugated hollow gold nanoshells (HAuNS) displaying an average diameter of $30 \mathrm{~nm}$ with cetuximab (CET), an antiepidermal growth factor receptor (EGFR) therapeutic antibody. CET-HAuNS selectively bound to cells overexpressing EGFR (A431 cells) that, upon irradiation with NIR laser, led to elevation of temperature, inducing cell death by photothermal ablation. Moreover, labeling of HAuNS with gamma emitter. ${ }^{97}$ In allowed monitoring tissue distribution of CET-HAuNS in vivo, revealing increased uptake in tumors expressing EGFR. ${ }^{91}$

SERS has also been used for tracking these multimodal NPs. ${ }^{69}$ Of particular interest is the work presented by Song et al ${ }^{98}$ in which an amphiphilic AuNP was coated with a Raman reporter BGLA (2-(4-(bis(4-(diethylamino)phenyl)(hydroxy) methyl)phenoxy)ethyl 5-(1,2-dithio-lan-3-yl)pentanoate). The particle was made stealth by PEG incorporation and $\mathrm{pH}-$ sensitive (due to PMMAVP grafts - hydrophobic copolymer of methyl methacrylate [MMA] and 4-vinylpyridine [4VP]). DOX was also included in this intricate system. Incorporation of HER2 antibody provided for specific targeting. Cellular binding, uptake, and intraorganelle disruption of the SERSencoded $\mathrm{pH}$-sensitive plasmonic vesicles were assessed. ${ }^{98}$ This unique system is an example of a promising platform for targeted theranostic applications of AuNPs.

\section{Theranostic nanomaterials: from the laboratory to the clinics?}

One of the first agents developed as a simultaneous targeting moiety and therapeutic agent was Herceptin ${ }^{\circledR}$ (trastuzumab; Hoffman-La Roche Ltd, Basel, Switzerland), a humanized antibody capable of targeting and blocking the overexpression of HER2 protein used for the treatment of HER2positive metastatic breast cancer. ${ }^{99,100}$ Similar approaches have followed, but most failed to deliver due to stand-alone limitations of these agents. ${ }^{101}$ Such failures have increased the costs of $\mathrm{R} \& \mathrm{D}$ without corresponding delivery to the market. As seen earlier, recent advances in nanotechnology have paved the way for the development of theranostics 


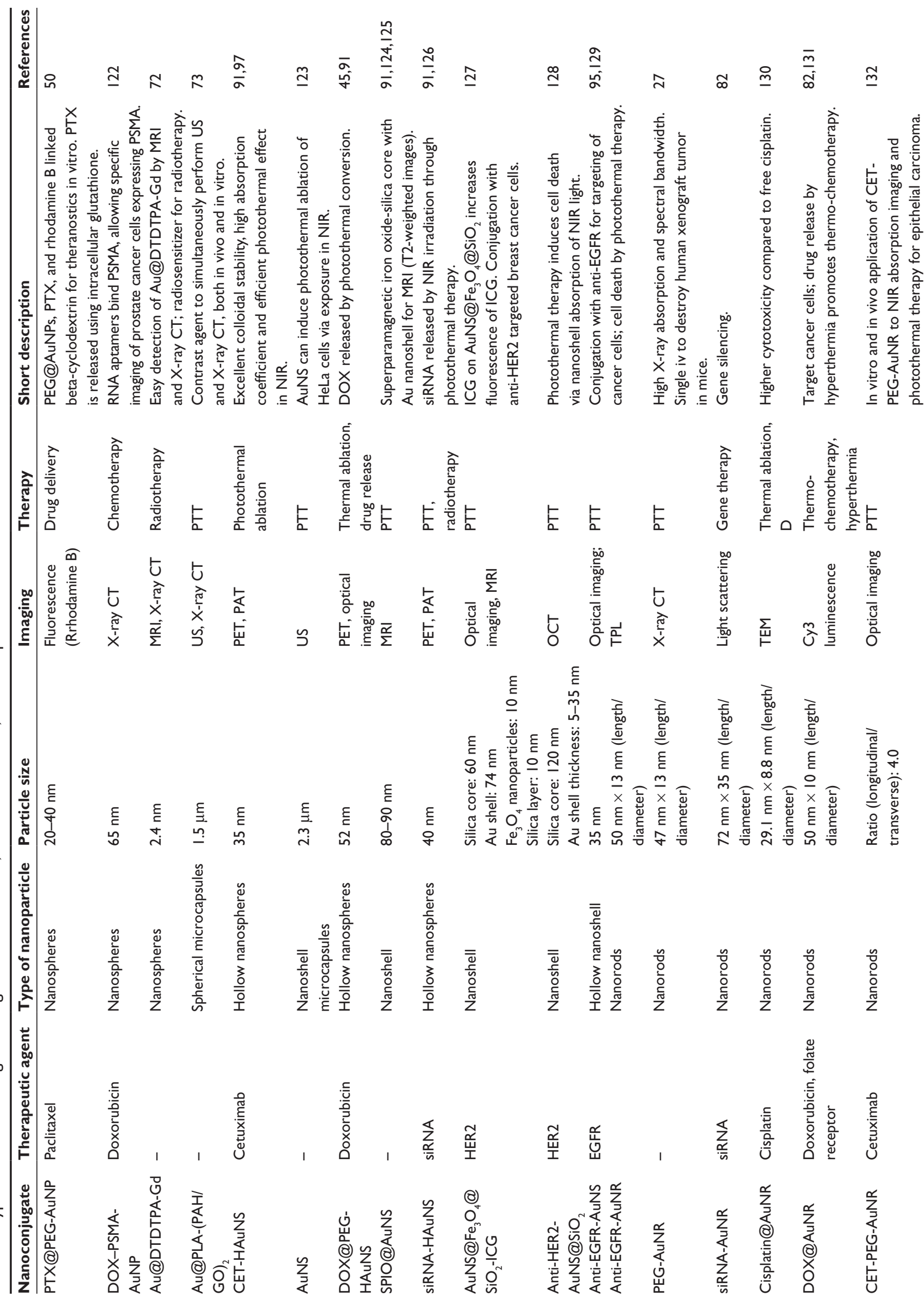



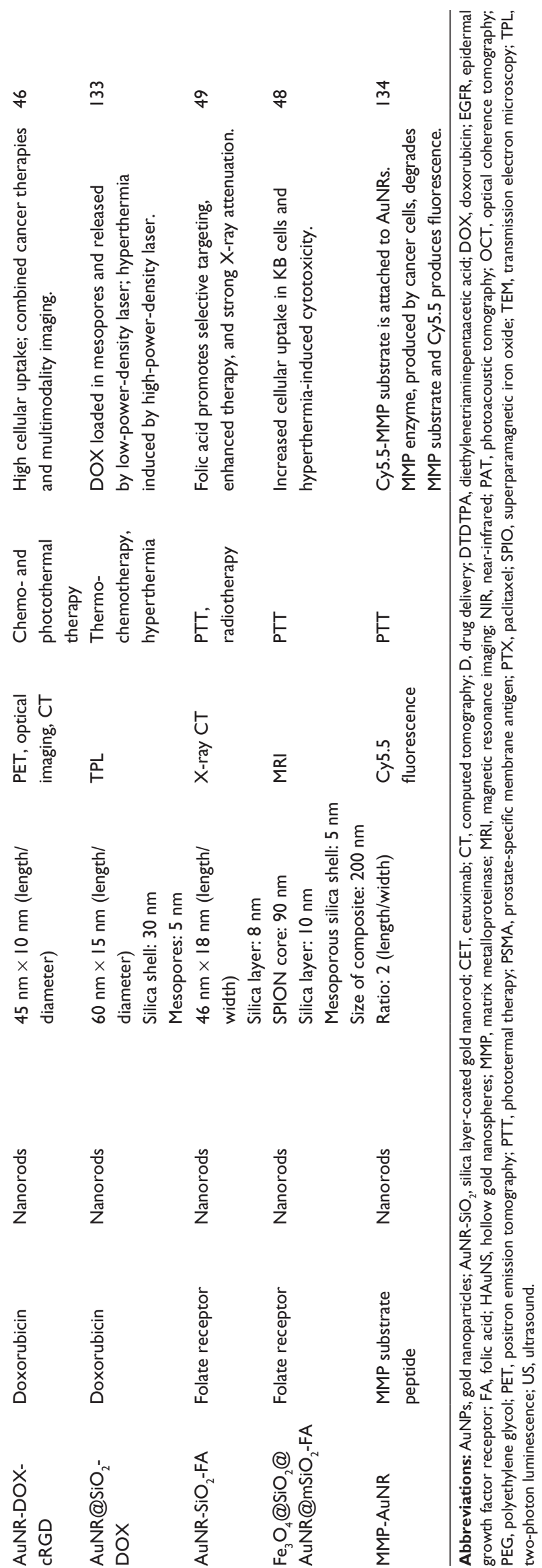

agents for a sustained, controlled, and targeted delivery of therapeutics coupled with the capability to follow in realtime distribution to tissues and organs, thus allowing therapy evaluation. ${ }^{63}$ These systems have been designed keeping in mind the selectivity for the most appropriate and effective therapy with fewer side effects. ${ }^{102,103}$ Despite the cumbersome task at hand, several nanosystems have been put forward for approval by the US Food and Drug Administration (FDA). Nanomedicines to make it to the market (worth US $\$ 33$ billion) include Doxil ${ }^{\circledR}$ (Janssen Biotech) $\left(\right.$ Caelyx ${ }^{\circledR}$ in Europe), Myocet ${ }^{\circledR}$ (Sopherion Therapeutics Inc, Princeton, NJ, USA), DaunoXome ${ }^{\circledR}$ (Galen US Inc, Souderton, PA, USA), DepoCyt ${ }^{\circledR}$ (Pacira Pharmaceuticals Inc, San Diego, CA, USA), Abraxane ${ }^{\circledR}$ (Celgene Corporation), Genexol-PM ${ }^{\circledR}$ (Samyang Biopharmaceuticals Corporation, Jongno-gu, Seoul, Korea), and Oncaspar ${ }^{\circledR}$ (Enzon Pharmaceuticals Inc, Bridgewater, NJ, USA)..$^{104}$

The overall nanomedicine market was evaluated in US\$53 billion (2009) and is expected to be worth a staggering $\$ 100$ billion in 2014. ${ }^{105}$ Several other FDA-approved nanomedicines have since reached the market, the last one to be Marqibo ${ }^{\circledR}$ (Spectrum Pharmaceuticals, Irvine, CA, USA) (FDA approval in 2012), which consists of a vincristine sulfate liposome injection for treatment of adult patients with Philadelphia chromosome-negative $\left(\mathrm{Ph}^{-}\right)$acute lymphoblastic leukemia in second or greater relapse or patients whose disease has still progressed despite two or more antileukemia therapies. Magnetic NPs are also making their way to clinical trials, and some formulations have been clinically approved for therapeutic applications and imaging (eg, Feridex ${ }^{\circledR}$ IV and Endorem ${ }^{\circledR}$ [Bayer Healthcare Pharmaceuticals] for liver and spleen imaging, Lumiren ${ }^{\circledR}$ (Guerbet SA, Villepinte, France) and Gastromark ${ }^{\circledR}$ (Advanced Magnetics Inc, Rochester, IN, USA) for bowel imaging). ${ }^{106}$ NanoTherm ${ }^{\circledR}$ (Magforce AG, Berlin, Germany), licenced in Europe since 2010 for the treatment of brain tumors, is an iron oxide NP administered to provide hyperthermia treatment localized to a tumor, sparing adjacent healthy tissue. ${ }^{107}$ A recent report shows that 247 nanomedicine products are approved or are in various stages of clinical study. ${ }^{108}$ Several more nanomedicines are to receive regulatory approval, but not all nanomedicines are available to the consumer. ${ }^{109}$ Almost 200 patents regarding NP-based drug delivery systems were registered between 2009 and 2012 in the World Intellectual Property Organization (WIPO), clearly indicating that these nanosystems may actually compete with conventional pharmaceuticals in the future. ${ }^{110}$

The ultimate goal in the R\&D of a nanomedicine is its successful translation from bench to bedside. As mentioned 
earlier, there are still significant challenges, such as 1) scalability, 2) batch-to-batch reproducibility, 3) lack of complete information concerning the interaction between nanotheranostics and tissues (in vivo - eg, internalization and biodistribution), 4) effective increase of therapeutic indexes, 5) reluctance of the pharmaceutical industry to invest in nanotheranostics, 6) regulatory issues and safety guidelines from authorities and agencies (eg, FDA and European Medicines Agency [EMA]) that have to be addressed before nanotheranostics exert their full impact on the clinics. ${ }^{106}$ The health benefits of nanotheranostics structures depend on their reproducibility and mostly differentiation from available products concerning improved efficacy, safety, and pharmacokinetics. ${ }^{106,111}$ Among these issues, research still needs to fully address nanotoxicology, in particular effects arising from the chronic administration. ${ }^{112}$ Furthermore, nanomedicine regulation still presents gaps, such as standardization, safety, and risk guidelines. Several key points need to be addressed in the near future before widespread use of nanomaterials, and nanotheranostics in particular: reference nanomaterials; uniformity of standards for and/or working definitions of nanomaterials; improvement to the current definitions of nanomaterial, for the purpose of regulation (namely, the difference between nanomaterial and nanoscale material); development of toxicology tests and physicochemical characterization studies for nanomaterials. These issues are key to ensure that nanomedicines and nanotheranostics platforms do not face commercialization bottlenecks due to lack of standards and parameters that support rational preclinical characterization. ${ }^{112}$ Nevertheless, FDA, in accordance with the Nanotechnology Characterization Laboratory (NCL), is gathering relevant information for the resolutions of some of the issues mentioned earlier. And EMA is ready to follow suit. By the conduction of transitional studies that will help nanomedicine developers to bring their academic work to the market, NCL gained the knowhow on which approaches are most effective in characterizing the properties of nanomedicines. For instance, NCL established more than 40 protocols to characterize NPs' physicochemical properties, in vitro immunogenicity, and cytotoxicity, together with the standard absorption, distribution, metabolism, excretion, and toxicity profiles, using animal models. ${ }^{113,114}$ Most of the reports available in the literature fail to deliver data on in vivo evaluation of nanomedicines and of nanotheranostic materials in particular. Existing data refer either to therapeutics or to diagnostics, leaving theranostics outside the scope of detailed testing. $3,102,115$

\section{Final remarks}

The use of nanomaterials for biosensing and diagnostics is making its way into the clinics, and industry is grasping the novelty and the amazing performance provided by these platforms and strategies to boost their market position. In what therapeutics is concerned, nanomedicines are entering a decisive phase of clinical trials, and plenty of nanoformulations and radical strategies and delivery platforms will not make through the strenuous process of validation and certification before settling into the clinical setting. What is more, these new nanoscale formulations have been strongly focused on anticancer therapeutics, where the risk-to-gain ratio clearly favors the development and assessment of nanomaterials.

Nanotheranostics is following these two vectors of development very close, taking advantage of hurdles and obstacles removed by the isolated counterparts. One should note that nanotheranostics is more than the addition of parts; a synergy between diagnostics and therapeutics is in place that could not be attained via the isolated effect of either strategy. Perhaps because of this great advantage, validation and characterization are even more complex and are still waiting for suitable tools to ascertain the real value in the clinics. Nanotheranostics is moving forward fast and focused, and one ought to be ready to make the best of these powerful tools in the coming years.

\section{Acknowledgments}

We acknowledge Fundação para a Ciência e a Tecnologia (FCT-MCE) for financial support CIGMH (PEst-OE/SAU/ UI0009/2011) and Project PTDC/BBB-NAN/1812/2012.

\section{Disclosure}

The authors report no conflicts of interest in this work.

\section{References}

1. Kelkar SS, Reineke TM. Theranostics: combining imaging and therapy. Bioconjugate Chem. 2011;22(10):1879-1903.

2. Mccarthy JR. Multifunctional agents for concurrent imaging and therapy in cardiovascular disease. Adv Drug Deliv Rev. 2011;62(11):1023-1030.

3. Wang L, Chuang MC, Ho JA. Nanotheranostics - a review of recent publications. Int J Nanomedicine. 2012;7:4679-4695.

4. Joh DY, Kao GD, Murty S, et al. Theranostic gold nanoparticles modified for durable systemic circulation effectively and safely enhance the radiation therapy of human sarcoma cells and tumors. Transl Oncol. 2013;6(6):722-731.

5. Luqmani YA. Mechanisms of drug resistance in cancer chemotherapy. Med Princ Pract. 2008;14(1):35-48.

6. Kaasgaard T, Andresen TL. Liposomal cancer therapy: exploiting tumor characteristics. Expert Opin Drug Del. 2010;7(2):225-243.

7. Massing U, Fuxius S. Liposomal formulations of anticancer drugs: selectivity and effectiveness. Drug Resist Updat. 2000;3(3):171-177.

8. Persidis A. Cancer multidrug resistance. Nature Biotechnol. 1999;17(1): 94-95. 
9. Ashworth A, Lord CJ, Reis-Filho JS. Genetic interactions in cancer progression and treatment. Cell. 2011;145(1):30-38.

10. Malam Y, Loizidou M, Seifalian AM. Liposomes and nanoparticles: nanosized vehicles for drug delivery in cancer. Trends Pharmacol Sci. 2009;30(11):592-599.

11. Bansal SS, Goel M, Aqil F, Vadhanam MV, Gupta RC. Advanced drug delivery systems of curcumin for cancer chemoprevention. Cancer Prev Res. 2011;4(8):1158-1171.

12. Cabral RM, Baptista PV. Anti-cancer precision theranostics: a focus on multifunctional gold nanoparticles. Expert Rev Mol Diagn. 2014; 14(8):1041-1052.

13. Ryu JH, Lee S, Son S, et al. Theranostic nanoparticles for future personalized medicine. J Control Release. 2014;190:477-484.

14. Conde J, Bao C, Cui D, Baptista PV, Tian F. Antibody-drug gold nanoantennas with Raman spectroscopic fingerprints for in vivo tumour theranostics. J Control Release. 2014;183:87-93.

15. Hu D, Sheng Z, Fang S, et al. Folate receptor-targeting gold nanoclusters as fluorescence enzyme mimetic nanoprobes for tumor molecular colocalization diagnosis. Theranostics. 2014;4(2):142-153.

16. Madhusudhan A, Reddy GB, Venkatesham M, et al. Efficient pH dependent drug delivery to target cancer cells by gold nanoparticles capped with carboxymethyl chitosan. Int J Mol Sci. 2014;15(5):8216-8234.

17. Wang X, Cai X, Hu J, et al. Glutathione-triggered "Off-On" release of anticancer drugs from dendrimer-encapsulated gold nanoparticles. J Am Chem Soc. 2013;(135):9805-9810.

18. Conde J, Tian F, Hernández Y, et al. In vivo tumor targeting via nanoparticle-mediated therapeutic siRNA coupled to inflammatory response in lung cancer mouse models. Biomaterials. 2013;34(31):7744-7753.

19. Conde J, Larguinho M, Cordeiro A. Gold-nanobeacons for gene therapy: evaluation of genotoxicity, cell toxicity and proteome profiling analysis. Nanotoxicology. 2014;8(5):521-532.

20. Xie J, Lee S, Chen X. Nanoparticle-based theranostic agents. Adv Drug Deliv Rev. 2010;62(11):1064-1079.

21. Draz M, Fang B, Zhang P, et al. Nanoparticle-mediated systemic delivery of siRNA for treatment of cancers and viral infections. Theranostics. 2014;4(9):1023-1030.

22. Dunne M, Zheng J, Rosenblat J, Jaffray DA, Allen C. APN/CD13targeting as a strategy to alter the tumor accumulation of liposomes. J Control Release. 2011;154(3):298-305.

23. Willmann JK, van Bruggen N, Dinkelborg LM, Gambhir SS. Molecular imaging in drug development. Nat Rev Drug Discov. 2008;7: 591-607.

24. Huang Y, He S, Cao W, Cai K, Liang XJ. Biomedical nanomaterials for imaging guided cancer therapy. Nanoscale. 2012;4:6135-6149.

25. Curry T, Kopelman R, Shilo M, Popovtzer R. Multifunctional theranostic gold nanoparticles for targeted $\mathrm{CT}$ imaging and photothermal therapy. Contrast Media Mol Imaging. 2014;9(1):53-61.

26. Cabral RM, Baptista PV. The chemistry and biology of gold nanoparticle-mediated photothermal therapy: promises and challenges. NanoLife. 2013;3(03).

27. von Maltzahn G, Park JH, Agrawal A, et al. Computationally guided photothermal tumor therapy using long-circulating gold nanorod antennas. Cancer Res. 2009;69(9):3892-3900.

28. Conde J, Rosa J, de la Fuente JM, Baptista PV. Gold-nanobeacons for simultaneous gene specific silencing and intracellular tracking of the silencing events. Biomaterials. 2013;34(10):2516-2523.

29. Maeda H, Nakamura H, Fang J. The EPR effect for macromolecular drug delivery to solid tumors: improvement of tumor uptake, lowering of systemic toxicity, and distinct tumor imaging in vivo. Adv Drug Deliv Rev. 2013;65(1):71-79.

30. Louie A. Multimodality imaging probes: design and challenges. Chem Rev. 2010;110(5):3146-3195.

31. Silberstein EB. Radioiodine: the classic theranostic agent. Semin Nucl Med. 2012;42(3):164-170.

32. Sperling R, Parak WJ. Surface modification, functionalization and bioconjugation of colloidal inorganic nanoparticles. Philos Trans A Math Phys Eng Sci. 2010;368(1915);1333-1383.
33. Pellegrino T, Kudera S, Liedl T, Muñoz Javier A, Manna L, Parak WJ. On the development of colloidal nanoparticles towards multifunctional structures and their possible use for biological applications. Small. 2005; 1(1):48-63.

34. Cho IH, Irudayaraj J. In-situ immuno-gold nanoparticle network ELISA biosensors for pathogen detection. Int J Food Microbiol. 2013;164(1): $70-75$.

35. Raoof M, Corr SJ, Kaluarachchi WD, et al. Stability of antibodyconjugated gold nanoparticles in the endo-lysosomal nanoenvironment: implications for non-invasive radiofrequency-based cancer therapy Nanomedicine. 2013;8(7):1096-1105.

36. Chávez JL, Leny JK, Witt S, Slusher GM, Hagen JA, Kelley-Loughnane N. Plasmonic aptamer-gold nanoparticle sensors for small molecule fingerprint identification. Analyst. 2014;139(23):6214-6222.

37. Conde J, Dias JT, Grazú V, Moros M, Baptista PV, de la Fuente JM. Revisiting 30 years of biofunctionalization and surface chemistry of inorganic nanoparticles for nanomedicine. Front Chem. 2014;2:48.

38. Alric C, Miladi I, Kryza D, et al. The biodistribution of gold nanoparticles designed for renal clearance. Nanoscale. 2013;5(13): 5930-5939.

39. Choi CHJ, Alabi C, Webster P, Davis ME. Mechanism of active targeting in solid tumors with transferrin-containing gold nanoparticles. Proc Natl Acad Sci U S A. 2010;107(3):1235-1240.

40. Maldiney T, Cyrille R, Seguin J, Wattier N, Bessodes M, Scherman D. Effect of core diameter, surface coating, and PEG chain length on the biodistribution of persistent luminescence nanoparticles in mice. ACS Nano. 2011;5(2):854-862.

41. Thobhani S, Attree S, Boyd R, et al. Bioconjugation and characterisation of gold colloid-labelled proteins. J Immunol Methods. 2010;356(1-2): $60-69$.

42. Conde J, Ambrosone A, Sanz V, et al. Design of multifunctional gold nanoparticles for in vitro and in vivo gene silencing. ACS Nano. 2012;6(9):8316-8324.

43. DeLong RK, Reynolds CM, Malcolm Y, Schaeffer A, Severs T, Wanekaya A. Functionalized gold nanoparticles for the binding, stabilization, and delivery of therapeutic DNA, RNA, and other biological macromolecules. Nanotechnol Sci Appl. 2010;3(1):53-63.

44. Kim CK, Ghosh P, Pagliuca C, Zhu ZJ, Menichetti S, Rotello VM. Entrapment of hydrophobic drugs in nanoparticle monolayers with efficient release into cancer cells. J Am Chem Soc. 2009;131(4): 1360-1361.

45. You J, Zhang G, Li C. Exceptionally high payload of doxorubicin in hollow gold nanospheres for near-infrared light-triggered drug release. ACS Nano. 2010;4(2):1033-1041.

46. Xiao Y, Hong H, Javadi A, et al. Gold nanorods conjugated with doxorubicin and cRGD for combined anticancer drug delivery and PET imaging. Theranostics. 2012;2(8):757-768.

47. Brown SD, Nativo P, Smith JA, et al. Gold nanoparticles for the improved anticancer drug delivery of the active component of oxaliplatin. J Am Chem Soc. 2010;132(13):4678-4684.

48. Wang D, Zhu X-M, Lee S-F, et al. Folate-conjugated $\mathrm{Fe}_{3} \mathrm{O}_{4} @ \mathrm{SiO}_{2} @$ goldnanorods@mesoporous $\mathrm{SiO}_{2}$ hybrid nanomaterial: a theranostic agent for magnetic resonance imaging and photothermal therapy J Mater Chem B. 2013;1(23):2934-2942.

49. Peng H, Bao L, Zhang C, et al. Folic acid-conjugated silica-modified gold nanorods for X-ray/CT imaging-guided dual-mode radiation and photo-thermal therapy. Biomaterials. 2011;32(36):9796-9809.

50. Heo D N, Yang DH, Moon HJ, Lee, et al. Gold nanoparticles surfacefunctionalized with paclitaxel drug and biotin receptor as theranostic agents for cancer therapy. Biomaterials. 2012;333:856-866.

51. Tabor C, Murali R, Mahmoud M, El-Sayed MA. On the use of plasmonic nanoparticle pairs as a plasmon ruler: the dependence of the near-field dipole plasmon coupling on nanoparticle size and shape. JPhys ChemA. 2009;113(10):1946-1953.

52. Pelton M, Aizpurua J, Bryant G. Metal-nanoparticle plasmonics. Laser Photonics Rev. 2008;2(3):136-159. 
53. Jain PK, Lee KS, El-Sayed IH, El-Sayed M. Calculated absorption and scattering properties of gold nanoparticles of different size, shape, and composition: applications in biological imaging and biomedicine. J Phys Chem B. 2006;110(14):7238-7248.

54. Ghosh SK, Pal T. Interparticle coupling effect on the surface plasmon resonance of gold nanoparticles: from theory to applications. Chem Rev. 2007;107(11):4797-4862.

55. Hu M, Chen J, Li ZY. Gold nanostructures: engineering their plasmonic properties for biomedical applications. Chem Soc Rev. 2006;35(11): 1084-1094.

56. Lin AY, Young JK, Nixon AV, Drezek RA. Synthesis of a quantum nanocrystal-gold nanoshell complex for near-infrared generated fluorescence and photothermal decay of luminescence. Nanoscale. 2014;6(18): 10701-10709.

57. Link S, El-Sayed M. Shape and size dependence of radiative, nonradiative and photothermal properties of gold nanocrystals. Int Rev Phys Chem. 2000;19(3):409-453.

58. Jain PK, Huang X, El-Sayed IH, El-Sayed M. Noble metals on the nanoscale: optical and photothermal properties and some applications in imaging, sensing, biology, and medicine. Acc Chem Res. 2008;41(12): $1578-1586$

59. Dreaden EC, Austin LA, Mackey MA, El-Sayed MA. Size matters: gold nanoparticles in targeted cancer drug delivery. Ther Deliv. 2012;3(4): 457-478.

60. Studwell AJ, Kotton DN. A shift from cell cultures to creatures: in vivo imaging of small animals in experimental regenerative medicine. Mol Ther. 2011;19(11):1933-1941.

61. Kim J, Yuanzhe P, Taeghwan H. Multifunctional nanostructured materials for multimodal imaging, and simultaneous imaging and therapy. Chem Soc Rev. 2009;38(2):372-390.

62. Marradi, M, Chiodo F, Penadés S. Glyconanoparticles as multifunctional and multimodal carbohydrate systems. Chem Soc Rev. 2013;42(11): 4728-4745.

63. Lammers T, Aime S, Hennink WE, Storm G, Kiessling F. Theranostic nanomedicine. Acc Chem Res. 2011;44(10):1029-1038.

64. Rosa JP, Lima JC, Baptista PV. Experimental photophysical characterization of fluorophores in the vicinity of gold nanoparticles. Nanotechnology. 2011;22(41):15202.

65. Kang KA, Wang J, Jasinski JB, Achilefu S. Fluorescence manipulation by gold nanoparticles: from complete quenching to extensive enhancement. J Nanobiotechnol. 2011;9:16.

66. Zhang J, Fu Y, Lakowicz JR. Enhanced Förster resonance energy transfer (FRET) on a single metal particle. J Phys Chem C. 2007;111:50-56.

67. Moskovits M. Surface-enhanced spectroscopy. Rev Mod Phys. 1985;57(3):783-828.

68. Xia L Wang J, Tong S, Liu G, Li J, Zhang H. Design and construction of a sensitive silver substrate for surface-enhanced Raman scattering spectroscopy. Vib Spectrosc. 2008;47(2):124-128.

69. Yigit MV, Medarova Z. In vivo and ex vivo applications of gold nanoparticles for biomedical SERS imaging. Am J Nucl Med Mol Imaging. 2012;2(2):232-241.

70. Lusic H, Grinstaff MW. X-ray-computed tomography contrast agents. Chem Rev. 2013;113(3):1641-1666.

71. Hasebroock KM, Serkova NJ. Toxicity of MRI and CT contrast agents. Expert Opin Drug Metab Toxicol. 2009;5(4):403-416.

72. Alric C, Taleb J, Duc G, et al. Gadolinium chelate coated gold nanoparticles as contrast agents for both X-ray computed tomography and magnetic resonance imaging. J Am Chem Soc. 2008;130(18):5908-5915.

73. Yushen J, Wang J, Ke H, Wang S, Dai Z. Graphene oxide modified PLA microcapsules containing gold nanoparticles for ultrasonic/CT bimodal imaging guided photothermal tumor therapy. Biomaterials. 2013;34(20):4794-4802.

74. Lee N, Choi SH, Hyeon T. Nano-sized CT contrast agents. Adv Mater. 2013;25(19):2641-2660.

75. Wang H, Zheng L, Peng C, et al. Computed tomography imaging of cancer cells using acetylated dendrimer-entrapped gold nanoparticles. Biomaterials. 2011;32(11):2979-2988.
76. Park JA, Kim HK, Kim JH, et al. Gold nanoparticles functionalized by gadolinium-DTPA conjugate of cysteine as a multimodal bioimaging agent. Bioorganic Med Chem Lett. 2010;20(7):2287-2291.

77. Wen S, Li K, Cai H, et al. Multifunctional dendrimer-entrapped gold nanoparticles for dual mode CT/MR imaging applications. Biomaterials. 2013;34(5):1570-1580.

78. Boisselier E, Astruc D. Gold nanoparticles in nanomedicine: preparations, imaging, diagnostics, therapies and toxicity. Chem Soc Rev. 2009;38(6):1759-1782.

79. Wang LV, Hu S. Photoacoustic tomography: in vivo imaging from organelles to organs. Science. 2012;335(6075):1458-1462.

80. Yao J, Wang LV. Breakthrough in photonics 2013: photoacoustic tomography in biomedicine. IEEE Photonics J. 2014;6(2).

81. Webb JA, Bardhan R. Emerging advances in nanomedicine with engineered gold nanostructures. Nanoscale. 2014;6(5):2502-2530.

82. Zhang, Z, Wang J, Chen C. Gold nanorods based platforms for lightmediated theranostics. Theranostics. 2013;3(3):223-238.

83. Mehrmohammadi M, Yoon SJ, Yeager D, Emelianov SY. Photoacoustic imaging for cancer detection and staging. Curr Mol Imaging. 2013;2(1): $89-105$.

84. Razansky D, Distel M, Vinegoni C, et al. Multispectral opto-acoustic tomography of deep-seated fluorescent proteins in vivo. Nat Photonics. 2009;3(7):412-417.

85. Cai X, Li L, Krumholz A, et al. Multi-scale molecular photoacoustic tomography of gene expression. PLoS One. 2012;7(8).

86. Laufer J, Jathoul A, Pule M, Beard P. In vitro characterization of genetically expressed absorbing proteins using photoacoustic spectroscopy. Biomed Opt Express. 2013;4(11):2477-2490.

87. Taruttis A, Lozano N, Nunes A, et al. siRNA liposome-gold nanorod vectors for multispectral optoacoustic tomography theranostics. Nanoscale. 2014;6(22)13451-13456.

88. Ghosh R, Singh LC, Shohet JM, Gunaratne PH. A gold nanoparticle platform for the delivery of functional microRNAs into cancer cells. Biomaterials. 2013;4(3):807-816.

89. Jung S, Nam J, Hwang S, et al. Theragnostic pH-sensitive gold nanoparticles for the selective surface enhanced Raman scattering and photothermal cancer therapy. Analytical Chem. 2013;85(16): 7674-7681.

90. Torchilin V. Multifunctional and stimuli-sensitive pharmaceutical nanocarriers. Eur J Pharm Biopharm. 2009;71(3):431-444.

91. Melancon MP, Zhou M, Li C. Cancer theranostics with near-infrared light-activatable multimodal nanoparticles. Acc Chem Res. 2011;44(10): 947-956.

92. Mayer G, Heckel A. Biologically active molecules with a "light switch". Angew Chem Int Ed. 2006;45(30):4900-4921.

93. Hironobu T, Niidome Y, Yamada S. Controlled release of plasmid DNA from gold nanorods induced by pulsed near-infrared light. Chem Commun. 2005; 17:2247-2249.

94. Melancon MP, Elliot AM, Huang Q, Li C. Near-infrared light modulated photothermal effect increases vascular perfusion and enhances polymeric drug delivery. J Control Release. 2011;156(2):265-272.

95. Kennedy LC, Bickford LR, Lewinski NA, et al. A new era for cancer treatment: gold-nanoparticle-mediated thermal therapies. Small. 2011;7(2):169-183.

96. Takahashi H, Niidome T, Nariai A, Niidome Y, Yamada S. Photothermal reshaping of gold nanorods prevents further cell death. Nanotechnology. 2006;17(17):4431-4435.

97. Melancon MP, Lu W, Yang Z, et al. In vitro and in vivo targeting of hollow gold nanoshells directed at epidermal growth factor receptor for photothermal ablation therapy. Mol Cancer Ther. 2008;7(6): $1730-1739$.

98. Song J, Zhou J, Duan H. Self-assembled plasmonic vesicles of SERSencoded amphiphilic gold nanoparticles for cancer cell targeting and traceable intracellular drug delivery. J Am Chem Soc. 2012;134(32): 13458-13469.

99. Brenner TL, Adams VR. First MAb approved for treatment of metastatic breast cancer. J Am Pharm Assoc (Wash). 1999;39(2):236-238. 
100. Paul SM, Mytelka DS, Dunwiddie CT, et al. How to improve R\&D productivity: the pharmaceutical industry's grand challenge. Nat Rev Drug Discov. 2010;9(3):203-214.

101. Muthu MS, Leong DT, Mei L, Feng SS. Nanotheranostics - application and further development of nanomedicine strategies for advanced theranostics. Theranostics. 2014;4(6):660-677.

102. Sakamoto JH, Van de Ven AL, Godin B, et al. Enabling individualized therapy through nanotechnology. Pharmacol Res. 2010;62(2): $57-89$.

103. Egusquiaguirre SP, Igartua M, Hernández RM, Pedraz JL. Nanoparticle delivery systems for cancer therapy: advances in clinical and preclinical research. Clin Transl Oncol. 2012;14:83-93.

104. BBC Research. Nanotechnology in Medical Applications: The Global Market. Wellesley, MA: BBC Research; Jan 2010. Available from: http://www.bccresearch.com/market-research/healthcare/ nanotechnology-medical-applications-hlc069a.html. Accessed January 3, 2015.

105. Ryan S, Brayden D. Progress in the delivery of nanoparticle constructs: toward clinical translation. Curr Opin Pharmacol. 2014;18: 120-128.

106. Mura S, Nicolas J, Couvreur P. Stimuli-responsive nanocarriers for drug delivery. Nat Mater. 2013;12:991-1003.

107. Etheridge ML, Campbell SA, Erdman AG, Haynes CL, Wolf SM, McCullough J. The big picture on nanomedicine: the state of investigational and approved nanomedicine products. Nanomedicine. 2013;9(1): $1-14$.

108. Bawa R. Nanopharmaceuticals: nanopharmaceuticals. Eur J Nanomed. 2010;3(1):34-40.

109. Martins P, Rosa D, Fernandes AR, Baptista PV. Nanoparticle drug delivery systems: recent patents and applications in nanomedicine. Recent Patents Nanomed. 2013;3(2):105-118.

110. Hawkins MJ, Soon-Shiong P, Desai N. Protein nanoparticles as drug carriers in clinical medicine. Adv Drug Deliv Rev. 2008;60(8): 876-885.

111. Monteiro-Riviere NA, Tran CL, editors. Nanotoxicology: Progress Toward Nanomedicine. 2nd ed. Boca Raton, FL: CRC Press; 2014

112. Bawa R. FDA and nanotech: baby steps lead to regulatory uncertainty. In: Bagchi D, Bagchi M, editors. Bio-Nanotechnology: A Revolution in Food, Biomedical and Health Sciences. 1st ed. Chichester, UK: Wiley; 2013:720-732.

113. Tinkle S, McNeil SE, Mühlebach S, et al. Nanomedicines: addressing the scientific and regulatory gap. Ann NY Acad Sci. 2014;1313(1): $35-56$.

114. Miller J. Beyond biotechnology: FDA regulation of nanomedicine. Columbia Sci Technol Law Rev. 2003;4:1-35.

115. Mura S, Couvreur P. Nanotheranostics for personalized medicine. Adv Drug Deliv Rev. 2012;64(13):1394-1416.

116. Manson J, Kumar D, Meenan B, Dixon D. Polyethylene glycol functionalized gold nanoparticles: the influence of capping density on stability in various media. Gold Bull. 2011;44(2):99-105.

117. Sanz V, Conde J, Hernández Y, Baptista P V, Ibarra MR, De La Fuente JM.Effect of PEGbiofunctional spacers andTATpeptide ondsRNA loading on gold nanoparticles. J Nanoparticle Res. 2012;14(6):917.
118. Park SY, Lee SM, Kim GB, Kim Y-P. Gold nanoparticle-based fluorescence quenching via metal coordination for assaying protease activity. Gold Bull. 2012;45(4):213-219.

119. Ravi S, Krishnamurthy VR, Caves JM, Haller CA, Chaikof EL. Maleimide-thiol coupling of a bioactive peptide to an elastin-like protein polymer. Acta Biomater. 2012;8(2):627-635.

120. Lee JS. Multiplexed detection of oligonucleotides with biobarcoded gold nanoparticle probes. Methods Mol Biol. 2011;726:17-31.

121. Hwu JR, Lin YS, Josephrajan T, et al. Targeted paclitaxel by conjugation to iron oxide and gold nanoparticles. JAm Chem Soc. 2009;131(1): 66-68.

122. Kim D, Jeong YY, Jon S. A drug-loaded aptamer-gold nanoparticle bioconjugate for combined CT imaging and therapy of prostate cancer ACS Nano. 2010;4(7):3689-3696.

123. Ke H, Wang J, Dai Z, et al. Gold-nanoshelled microcapsules: a theranostic agent for ultrasound contrast imaging and photothermal therapy. Angew Chem. 2011;123(13):3073-3077.

124. Melancon MP, Elliot A, Ji X. Theranostics with multifunctional magnetic gold nanoshells: photothermal therapy and $\mathrm{t} 2 *$ magnetic resonance imaging. Invest Radiol. 2011;46(2):132-140.

125. Ji X, Shao R, Elliot A, et al. Bifunctional gold nanoshells with a superparamagnetic iron oxide-silica core suitable for both MR imaging and photothermal therapy. J Phys Chem C. 2007;111(17):6245-6251.

126. Lu W, Zhang G, Zhang R, et al. Tumor site-specific silencing of NF- $\mathrm{KB}$ p65 by targeted hollow gold nanosphere-mediated photothermal transfection. Cancer Res. 2010;70(8):3177-3188.

127. Bardhan R, Chen W, Perez-Torres C, et al. Nanoshells with targeted simultaneous enhancement of magnetic and optical imaging and photothermal therapeutic response. Adv Funct Mater. 2009;19(24): 3901-3909.

128. Loo C, Lin A, Hirsch L, et al. Nanoshell-enabled photonics-based imaging and therapy of cancer. Technol Cancer Res Treat. 2004;3(1): 33-40.

129. Huang X, El-Sayed M. Gold nanoparticles: interesting optical properties and recent applications in cancer diagnostics and therapy. $J A d v$ Res. 2007;681-693.

130. Hauck ST, Jennings TL, Yatsenko T, Kumaradas JC, Chan W. Enhancing the toxicity of cancer chemotherapeutics with gold nanorod hyperthermia. Adv Mater. 2008;20(20):3832-3838.

131. Xiao Z, Ji C, Shi J, et al. DNA self-assembly of targeted near-infraredresponsive gold nanoparticles for cancer thermo-chemotherapy. Angew Chem. 2012;124(47):12023-12027.

132. Choi J, Yang J, Bang D, et al. Targetable gold nanorods for epithelial cancer therapy guided by near-IR absorption imaging. Small. 2012;8(5):746-753.

133. Zhang Z, Wang L, Wang J, et al. Mesoporous silica-coated gold nanorods as a light-mediated multifunctional theranostic platform for cancer treatment. Adv Mater. 2012;24(11):1418-1423.

134. Yi DK, Sun I-C, Ryu J, et al. Matrix metalloproteinase sensitive gold nanorod for simultaneous bioimaging and photothermal therapy of cancer. Bioconjugate Chem. 2010;21(12):2173-2177.
Nanobiosensors in Disease Diagnosis

\section{Publish your work in this journal}

Nanobiosensors in Disease Diagnosis is an international, peer-reviewed, open access journal publishing original research, reports, reviews and commentaries including but not confined to: Diagnosis of diseases including cancer, cardiovascular, infectious diseases; Molecular modeling in diagnosis; Enzyme and membrane technologies; and quantum

\section{Dovepress}

dot fluorescence technologies for monitoring toxins and pathogens. The manuscript management system is completely online and includes a very quick and fair peer-review system, which is all easy to use. Visit http://www.dovepress.com/testimonials.php to read real quotes from published authors. 